\title{
Enhancement of basic fibroblast growth factor-stimulated VEGF synthesis by Wnt3a in osteoblasts
}

\author{
HARUHIKO TOKUDA $^{1,2}$, SEIJI ADACHI ${ }^{2}$, RIE MATSUSHIMA-NISHIWAKI ${ }^{2}$, KENJI KATO $^{2,3}$, \\ HIDEO NATSUME ${ }^{2,3}$, TAKANOBU OTSUKA ${ }^{3}$ and OSAMU KOZAWA ${ }^{2}$ \\ ${ }^{1}$ Department of Clinical Laboratory, National Center for Geriatrics and Gerontology, Obu 474-8511; ${ }^{2}$ Department \\ of Pharmacology, Gifu University Graduate School of Medicine, Gifu 501-1194; ${ }^{3}$ Department of Orthopedic \\ Surgery, Nagoya City University Graduate School of Medical Sciences, Nagoya 467-8601, Japan
}

Received December 9, 2010; Accepted January 24, 2011

DOI: $10.3892 / \mathrm{ijmm} .2011 .644$

\begin{abstract}
It is currently recognized that the Wnt signaling pathway regulates bone mass. We have previously reported that the basic fibroblast growth factor (FGF-2) stimulates the synthesis of the vascular endothelial growth factor (VEGF) at least in part via the p44/p42 mitogen-activated protein (MAP) kinase and the stress-activated protein kinase (SAPK)/c-Jun $\mathrm{N}$-terminal kinase (JNK) in osteoblast-like MC3T3-E1 cells. In the present study, we investigated the effect of Wnt3a on FGF-2-stimulated VEGF synthesis in MC3T3-E1 cells. Wnt3a significantly augmented the FGF-2-stimulated VEGF release in a dose-dependent manner in the range between 1 and $30 \mathrm{ng} / \mathrm{ml}$. Lithium chloride and SB216763, inhibitors of glycogen synthase kinase $3 \beta$ (GSK3 $\beta$ ), enhanced the FGF-2-stimulated VEGF release. Wnt3a did not affect the phosphorylation of the $\mathrm{p} 44 / \mathrm{p} 42$ MAP kinase, SAPK/JNK, Akt, p38 MAP kinase or the p70 S6 kinase induced by FGF-2. Wnt3a and SB216763 increased the levels of VEGF mRNA expression induced by FGF-2. These results strongly suggest that Wnt3a enhances VEGF synthesis stimulated by FGF-2 via activation of the canonical Wnt/ $\beta$ catenin pathway in osteoblasts.
\end{abstract}

\section{Introduction}

Vascular endothelial growth factor (VEGF) is an angiogenic growth factor displaying high specificity for vascular endothelial cells (1). VEGF, which is produced and secreted from a variety of cell types, increases capillary permeability and stimulates proliferation of endothelial cells (1). Metabolism of the skeleton consisting of bone formation and bone resorption are respectively regulated by osteoblasts and osteoclasts (2). Osteoblasts and osteoclasts are continu-

Correspondence to: Dr Haruhiko Tokuda, Department of Clinical Laboratory, National Center for Geriatrics and Gerontology, Obu 474-8511, Japan

E-mail: tokuda@ncgg.go.jp

Key words: Wnt, fibroblast growth factor, vascular endothelial growth factor, $\beta$-catenin, osteoblast ally implicated in bone regeneration and repair (2). The microvasculature is provided by capillary endothelial cells during bone remodeling. It is generally recognized that the activities of osteoblasts, osteoclasts and capillary endothelial cells are closely coordinated and regulate bone metabolism (3). These functional cells are considered to influence one another via humoral factors as well as by direct cell-to-cell contact. As for bone metabolism, it has been reported that inactivation of VEGF causes complete suppression of blood vessel invasion concomitant with impaired trabecular bone formation and expansion of hypertrophic chondrocyte zone in the mouse tibial epiphyseal growth plate (4). Accumulating evidence suggests that osteoblasts among bone cells produce and secrete VEGF in response to various physiological agents such as insulin-like growth factor-I and bone morphogenetic protein (4). Therefore, it is speculated that VEGF secreted from osteoblasts may play a pivotal role in the regulation of bone metabolism $(3,4)$.

On the other hand, basic fibroblast growth factor (FGF-2) is synthesized in osteoblasts and embedded in the bone matrix (5). FGF-2 expression in osteoblasts is detected during fracture repair (6). Therefore, it is recognized that FGF-2 may play a pivotal role in fracture healing, bone remodeling and osteogenesis (7). It has been reported that FGF-2 stimulates the expression of VEGF in osteoblasts (8). In our previous studies $(9,10)$, we have shown that in osteoblast-like MC3T3-E1 cells, FGF-2 stimulates VEGF release, and that among the mitogen-activated protein (MAP) kinase superfamily (11), the p44/p42 MAP kinase and the stress-activated protein kinase (SAPK)/c-Jun N-terminal kinase (JNK) play important roles as positive regulators in the VEGF release. Based on these findings, the VEGF secretion induced by FGF-2 from osteoblasts may couple angiogenesis to bone formation by adjusting the angiogenic response to osteoblastic activity (4). However, the exact regulatory mechanism behind VEGF synthesis in osteoblasts remains to be clarified.

It is well known that Wnt-ligands, secreted glycoproteins, regulate early embryonic development, cell proliferation, differentiation and survival $(12,13) . \beta$-catenin is one of the intracellular downstream molecules in the Wnt pathway. In the absence of the canonical Wnt signal, $\beta$-catenin is targeted for degradation by glycogen synthase kinase $3 \beta$ (GSK3 $\beta$ ) 
and the accumulation in the nucleus is inhibited (12). Wnts bind to Frizzled receptors and the low-density lipoprotein receptor-related protein 5 or 6 (LRP5/6) in vertebrates $(13,14)$. This complex induces the phosphorylation and inactivation of GSK $3 \beta$, resulting in the accumulation of $\beta$-catenin in the nucleus (12). Nuclear $\beta$-catenin binds the transcription of downstream target genes via $\mathrm{T}$ cell factors and lymphoid enhancer-binding factor, and induces target gene expression $(13,14)$. In bone tissue, osteoblasts produce Wnt proteins (14). Evidence is accumulating that the Wnt signaling pathway plays a crucial role in bone metabolism especially bone formation (14-16). It has been shown that loss or gain of function mutations in LRP5 in bone tissue are linked with the osteoporosis-pseudoglioma syndrome, or a high bone density syndrome, respectively in humans (14). Moreover, genetic deletion of $\beta$-catenin from early osteoprogenitors results in lack of mature osteoblasts in the mouse embryo (14), whereas forced activation of $\beta$-catenin greatly enhances osteogenesis (17). However, the exact roles of the Wnt $/ \beta$-catenin signaling pathway in osteoblasts have not yet been clarified.

In the present study, we investigated the effect of Wnt3a on the FGF-2-stimulated VEGF synthesis in osteoblast-like MC3T3-E1 cells since it has been shown that Wnt3a amongst the Wnt-ligands mainly activates the canonical pathway in osteoblasts (18). We show that Wnt3a up-regulates FGF-2stimulated VEGF synthesis via the canonical Wnt/ $\beta$-catenin pathway in MC3T3-E1 cells.

\section{Materials and methods}

Materials. FGF-2, Wnt3a and the mouse VEGF enzyme immunosorbent assay (ELISA) kit were purchased from R\&D Systems, Inc. (Minneapolis, MN). Lithium chloride ( $\mathrm{LiCl}$ ) was purchased from Nacalai Tesque, Inc. (Kyoto, Japan). The GSK3 $\beta$ inhibitor, SB216763 was obtained from Calbiochem-Novabiochem Co. (La Jolla, CA). The following antibodies were purchased from Cell Signaling Technology, Inc. (Beverly, MA): phospho-specific p44/p42 MAP kinase, p44/p42 MAP kinase, phospho-specific SAPK/JNK, SAPK/ JNK, phospho-specific Akt, Akt, phospho-specific p38 MAP kinase, p38 MAP kinase, phospho-specific p70 S6 kinase and the p70 S6 kinase antibodies. The ECL Western blotting detection system was purchased from GE Healthcare UK Ltd. (Buckinghamshire, UK). SB216763 was dissolved in dimethyl sulfoxide. Other materials and chemicals were obtained from commercial sources. The maximum concentration of dimethyl sulfoxide was $0.1 \%$, which did not affect the assay for ELISA or Western blot analysis.

Cell culture. The cloned osteoblast-like MC3T3-E1 cells, which have been derived from newborn mouse calvaria (19), were maintained as previously described (20). Briefly, the cells were cultured in $\alpha$-minimum essential medium $(\alpha-M E M)$ containing $10 \%$ fetal calf serum (FCS) at $37^{\circ} \mathrm{C}$ in a humidified atmosphere of $5 \% \mathrm{CO}_{2} / 95 \%$ air. The cells were seeded into $35-\mathrm{mm}$ diameter dishes $\left(5 \times 10^{4} / \mathrm{dish}\right)$ or $90-\mathrm{mm}$ diameter dishes $\left(20 \times 10^{4} /\right.$ dish) in $\alpha$-MEM containing $10 \%$ FCS. After 5 days, the medium was exchanged to $\alpha$-MEM containing $0.3 \%$ FCS and incubated for $48 \mathrm{~h}$. The cells were then used for respective experiments.
Assay for VEGF. The cultured cells were pre-treated with various doses of Wnt3a, $\mathrm{LiCl}$ or SB216763 for $60 \mathrm{~min}$ and then stimulated by $50 \mathrm{ng} / \mathrm{ml} \mathrm{FGF-2}$ or vehicle in $\alpha$-MEM containing $0.3 \%$ FCS for the indicated periods. The conditioned medium was collected, and VEGF in the medium was then measured by a mouse VEGF ELISA kit according to the manufacturer's protocol. The absorbance of enzyme immunoassay samples was measured at $450 \mathrm{~nm}$ with the EL 340 Bio Kinetic Reader (Bio-Tek Instruments, Inc., Winooski, VT).

Real-time RT-PCR. The cultured cells were pre-treated with $30 \mathrm{ng} / \mathrm{ml} \mathrm{Wnt3a}, 10 \mu \mathrm{M}$ SB216763 or vehicle for $60 \mathrm{~min}$, and then stimulated by $50 \mathrm{ng} / \mathrm{ml} \mathrm{FGF-2} \mathrm{in} \alpha$-MEM containing $0.3 \%$ FCS for the indicated periods. Total-RNA was isolated and transcribed into complementary DNA using TRIzol reagent (Invitrogen Corp., Carlsbad, CA) and Omniscript Reverse Transcriptase kit (Qiagen Inc., Valencia, CA), respectively. Real-time RT-PCR was performed using a LightCycler system in capillaries and FastStart DNA Master SYBR-Green I provided with the kit (Roche Diagnostics, Basel, Switzerland). Sense and antisense primers were synthesized based on the report of Simpson et al for mouse GAPDH mRNA (21). Sense and antisense primers for mouse VEGF mRNA were purchased from Takara Bio Inc. (Tokyo, Japan) (primer set ID: MA039013). The amplified products were determined by melting curve analysis and agarose electrophoresis. VEGF mRNA levels were normalized to those of GAPDH mRNA.

Western blot analysis. Western blot analysis was performed as previously described (22). In brief, the cultured cells were pre-treated with various doses of Wnt3a for $60 \mathrm{~min}$ and then stimulated by $30 \mathrm{ng} / \mathrm{ml} \mathrm{FGF-2}$ or vehicle in $\alpha$-MEM containing $0.3 \%$ FCS for the indicated periods. The cells were washed twice with phosphate-buffered saline and then lysed, homogenized and sonicated in a lysis buffer containing $62.5 \mathrm{mM}$ Tris-HCl, $\mathrm{pH} 6.8 ; 3 \%$ sodium dodecyl sulfate (SDS); $50 \mathrm{mM}$ dithiothreitol, and $10 \%$ glycerol. SDS-polyacrylamide gel electrophoresis (PAGE) was performed according to Laemmli (23) on $10 \%$ polyacrylamide gel. The protein $(20 \mu \mathrm{g})$ was fractionated and transferred onto an Immun-Blot PVDF Membrane (Bio-Rad, Hercules, CA). Membranes were blocked with 5\% fat-free dry milk in Tris-buffered salineTween (TBS-T; 20 mM Tris-HCl, pH 7.6, $137 \mathrm{mM} \mathrm{NaCl}, 0.1 \%$ Tween-20) for $2 \mathrm{~h}$ before incubation with the primary antibodies. The following rabbit polyclonal antibodies were used: phospho-specific p44/p42 MAP kinase, p44/p42 MAP kinase, phospho-specific SAPK/JNK, SAPK/JNK, phospho-specific Akt, Akt, phospho-specific p38 MAP kinase, p38 MAP kinase, phospho-specific p70 S6 kinase or the p70 S6 kinase primary antibodies. Peroxidase-labeled antibodies raised in goat against rabbit IgG were used as secondary antibodies. The primary and secondary antibodies were diluted at 1:1000 with 5\% fat-free dry milk in TBS-T. Peroxidase activity on the membrane was visualized on X-ray film by means of the ECL Western blotting detection system.

Statistical analysis. The data were analyzed by ANOVA followed by the Bonferroni method for multiple comparisons between pairs, and a $p<0.05$ was considered significant. All data are presented as the mean \pm SEM of triplicate determina- 


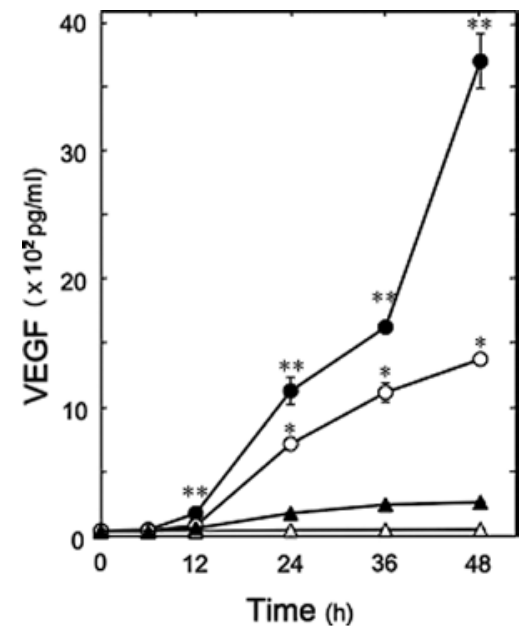

Figure 1. Effect of Wnt3a on the FGF-2-stimulated VEGF release in MC3T3-E1 cells. The cultured cells were pre-treated with $30 \mathrm{ng} / \mathrm{ml} \mathrm{Wnt3a}$

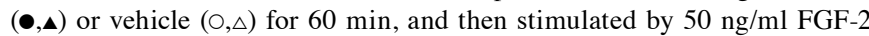
(circles) or vehicle (triangles) for the indicated periods. Each value represents the mean \pm SEM of triplicate determinations. Similar results were obtained with two additional and different cell preparations. ${ }^{*} p<0.05$, compared to the value of the control; ${ }^{* *} \mathrm{p}<0.05$, compared to the value of FGF-2 alone.



Figure 2. Dose-dependent effect of Wnt3a on the FGF-2-stimulated VEGF release in MC3T3-E1 cells. The cultured cells were pre-treated with various doses of Wnt3a for $60 \mathrm{~min}$, and then stimulated by $50 \mathrm{ng} / \mathrm{ml} \mathrm{FGF-2} \mathrm{( \bullet )} \mathrm{or}$ vehicle (O) for $48 \mathrm{~h}$. Each value represents the mean \pm SEM of triplicate determinations. Similar results were obtained with two additional and different cell preparations. ${ }^{*} \mathrm{p}<0.05$, compared to the value of FGF-2 alone.

tions. Each experiment was repeated three times with similar results.

\section{Results}

Effect of Wnt3a on the FGF-2-stimulated VEGF release in osteoblast-like MC3T3-E1 cells. We previously demonstrated that the maximum effect of FGF-2 on VEGF release was observed at $48 \mathrm{~h}$ after the stimulation (9). In the present study, we first examined the effect of Wnt3a on VEGF release induced by FGF-2 in osteoblast-like MC3T3-E1 cells. Wnt3a significantly enhanced the FGF-2-induced VEGF release in a time-dependent manner (Fig. 1). The amplifying effect of

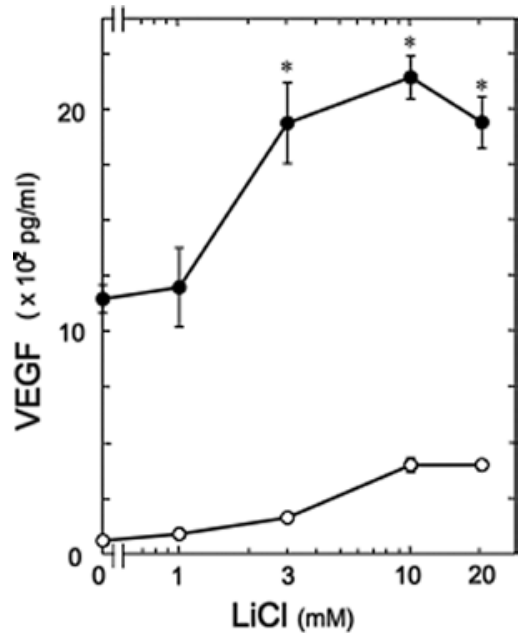

Figure 3. Effect of $\mathrm{LiCl}$ on the FGF-2-stimulated VEGF release in MC3T3-E1 cells. The cultured cells were pre-treated with various doses of $\mathrm{LiCl}$ for $60 \mathrm{~min}$, and then stimulated by $50 \mathrm{ng} / \mathrm{ml} \mathrm{FGF}-2(\bullet)$ or vehicle (O) for $48 \mathrm{~h}$. Each value represents the mean \pm SEM of triplicate determinations. Similar results were obtained with two additional and different cell preparations. ${ }^{*}$ p $<0.05$, compared to the value of FGF-2 alone.

Wnt3a on the FGF-2-stimulated VEGF release was dosedependent in the range between 1 and $30 \mathrm{ng} / \mathrm{ml}$ (Fig. 2). The maximum effect of Wnt3a was observed at $10 \mathrm{ng} / \mathrm{ml}$, which caused an $\sim 130 \%$ amplification of the FGF-2 effect.

Effect of LiCl or SB216763 on the FGF-2-stimulated VEGF release in MC3T3-E1 cells. In the canonical Wnt/ $\beta$-catenin pathway, the signaling by Wnt suppresses the GSK3 $\beta$ activity that phosphorylates $\beta$-catenin and induces the degradation of $\beta$-catenin $(12,13)$. It has recently been reported that $\beta$-catenin accumulation is stimulated by lithium, an inhibitor of GSK3 $\beta$ (24), as well as Wnt in pre-osteoblast CIMC-4 cells (25). Therefore, we next examined the effect of $\mathrm{LiCl}$ on the FGF-2stimulated VEGF release in osteoblast-like MC3T3-E1 cells. $\mathrm{LiCl}$ significantly potentiated the FGF-2-induced net increase of VEGF release (Fig. 3). The amplification by $\mathrm{LiCl}$ of the VEGF release was dose-dependent in the range between 1 and $20 \mathrm{mM}$. The maximum effect of $\mathrm{LiCl}$ was observed at $10 \mathrm{mM}$, which caused an $\sim 60 \%$ enhancement in the FGF-2effect (Fig. 3).

In addition, we further examined the effect of SB216763, another inhibitor of GSK3 $\beta$ (26), on the FGF-2-induced VEGF release. SB216763 as well as $\mathrm{LiCl}$ significantly enhanced the VEGF release in a dose-dependent manner between 0.3 and $10 \mu \mathrm{M}$ (Fig. 4). SB216763 (10 mM) resulted in an $\sim 230 \%$ enhancement of the FGF-2 effect (Fig. 4).

Effect of Wnt3a or SB216763 on the FGF-2-induced expression levels of VEGF mRNA in MC3T3-E1 cells. It has been reported that FGF-2 induces the VEGF expression in osteoblasts (8). In order to investigate whether the amplifying effect of Wnt3a on FGF-2-stimulated VEGF release is mediated through transcriptional event or not in MC3T3-E1 cells, we furthermore examined the effect of Wnt3a or SB216763 on the FGF-2-induced VEGF mRNA expression. We confirmed that FGF-2 time-dependently increased VEGF mRNA expression 


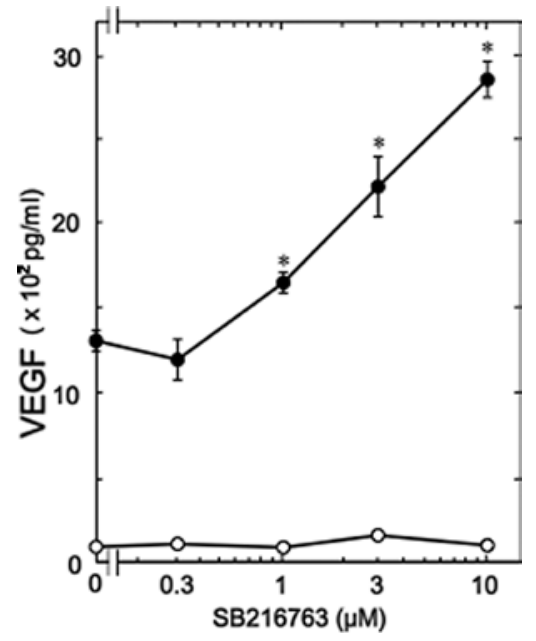

Figure 4. Effect of SB216763 on the FGF-2-stimulated VEGF release in MC3T3-E1 cells. The cultured cells were pre-treated with various doses of SB216763 for $60 \mathrm{~min}$, and then stimulated by $50 \mathrm{ng} / \mathrm{ml} \mathrm{FGF-2} \mathrm{( \bullet )} \mathrm{or} \mathrm{vehicle}$ (O) for $48 \mathrm{~h}$. Each value represents the mean \pm SEM of triplicate determinations. Similar results were obtained with two additional and different cell preparations. " $\mathrm{p}<0.05$, compared to the value of FGF- 2 alone.

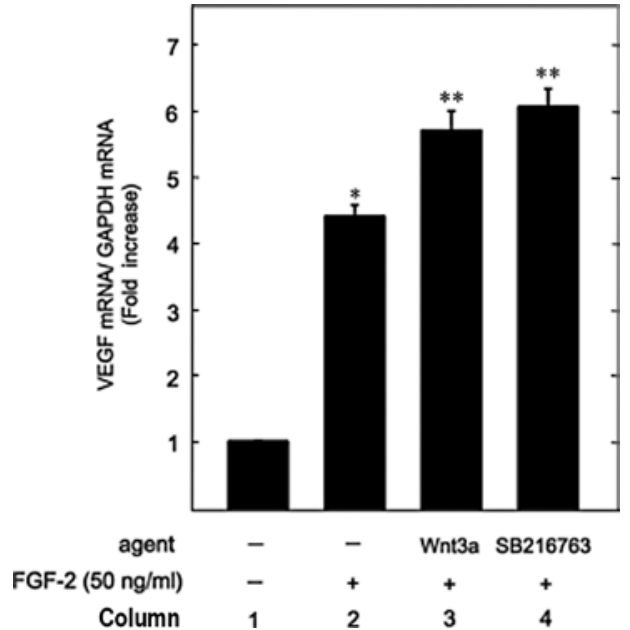

Figure 5. Effect of Wnt3a or SB216763 on the FGF-2-induced expression levels of VEGF mRNA in MC3T3-E1 cells. The cultured cells were pretreated with $30 \mathrm{ng} / \mathrm{ml} \mathrm{Wnt} 3 \mathrm{a}, 10 \mu \mathrm{M} \mathrm{SB} 216763$ or vehicle for $60 \mathrm{~min}$, and then stimulated by $50 \mathrm{ng} / \mathrm{ml} \mathrm{FGF-2} \mathrm{for} 12 \mathrm{~h}$. The respective total RNA were then isolated and quantified by real-time RT-PCR. Results were standardized for the value of control. Each value represents the mean \pm SEM of triplicate determinations. Similar results were obtained with two additional and different cell preparations. " $\mathrm{p}<0.05$, compared to the value of the control; ${ }^{* *} \mathrm{p}<0.05$, compared to the value of FGF-2 alone.

levels in these cells. Wnt3a (30 ng/ml) significantly augmented the FGF-2-induced VEGF mRNA expression levels (Fig. 5, column 3). SB216763 $(10 \mu \mathrm{M})$ in addition to Wnt3a enhanced the FGF-2-induced VEGF mRNA expression levels (Fig. 5, column 4).

Effects of Wnt3a on the phosphorylation of p44/p42 MAP kinase, SAPK/JNK, Akt, p38 MAP and p70 S6 kinase induced by FGF-2 in MC3T3-El cells. We have previously reported that FGF-2 stimulates the release of VEGF at least in part via the p44/p42 MAP kinase and the SAPK/JNK in osteoblastlike MC3T3-E1 cells $(9,10)$. In order to investigate whether
A

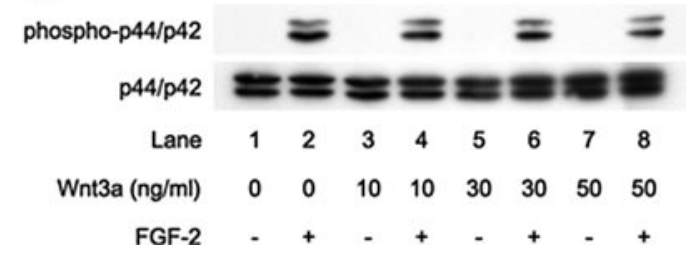

B

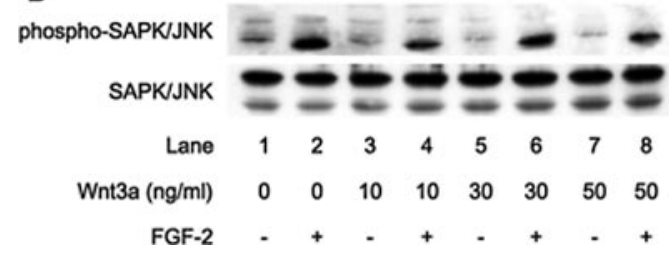

C

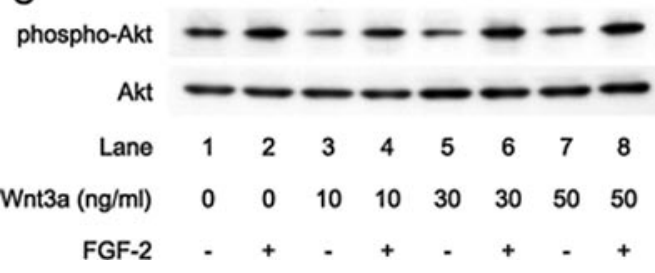

D

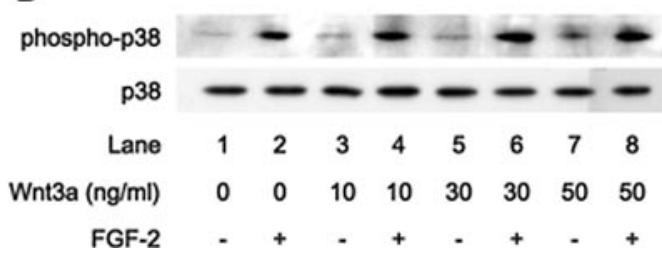

E

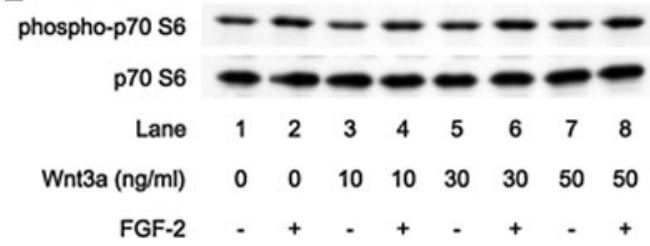

Figure 6. Effects of Wnt3a on the FGF-2-induced phosphorylation of the p44/p42 MAP kinase (A), SAPK/JNK (B), Akt (C), p38 MAP kinase (D) and p70 S6 kinase (E) in MC3T3-E1 cells. The cultured cells were pre-treated with various doses of Wnt3a for $60 \mathrm{~min}$, and then stimulated with $50 \mathrm{ng} / \mathrm{ml}$ FGF-2 or vehicle for $120 \mathrm{~min}$. The cell extracts were then subjected to SDS-PAGE with subsequent Western blot analysis with antibodies against phospho-specific p44/p42 MAP kinase, p44/p42 MAP kinase, phospho-specific SAPK/JNK, SAPK/JNK, phospho-specific Akt, Akt, phospho-specific p38 MAP kinase, p38 MAP kinase, phospho-specific p70 S6 kinase or p70 S6 kinase antibodies. Similar results were obtained with two additional and different cell preparations.

the amplifying effect of Wnt3a on the VEGF synthesis is dependent on the activation of $\mathrm{p} 44 / \mathrm{p} 42$ MAP kinase or the SAPK/JNK pathway in these cells, we examined the effect of Wnt3a on the FGF-2-induced phosphorylation of p44/p42 MAP kinase and SAPK/JNK. Wnt3a (10-50 ng/ml) failed to strengthen the FGF-2-induced phosphorylation levels of p44/p42 MAP kinase or the SAPK/JNK (Fig. 6A and B).

In our previous studies $(9,27,28)$, we have demonstrated that Akt, p38 MAP kinase and p70 S6 kinase function as negative regulators in the FGF-2-stimulated VEGF release 
in osteoblast-like MC3T3-E1 cells and limit the release of VEGF. Therefore, we next examined the effects of Wnt3a on FGF-2-induced phosphorylation of Akt, p38 MAP kinase or p70 S6 kinase. However, Wnt3a failed to suppress the FGF-2stimulated phosphorylation levels of Akt (Fig. 6C), p38 MAP kinase (Fig. 6D) or p70 S6 kinase (Fig. 6E).

\section{Discussion}

In the present study, we demonstrated that Wnt3a significantly potentiated VEGF release stimulated by FGF-2 in osteoblast-like MC3T3-E1 cells. It is well recognized that the canonical pathway is a major signaling pathway of Wnt (12). Wnt stimulation suppresses the activity of GSK $3 \beta$, a protein kinase which induces degradation of $\beta$-catenin, and its subsequent accumulation in the nucleus (12). It has been reported that Wnt3a increases $\beta$-catenin levels in osteoblasts (29). We confirmed that Wnt3a markedly enhanced the protein levels of $\beta$-catenin in a time-dependent manner in the osteoblast-like, MC3T3-E1 cells. We next demonstrated that $\mathrm{LiCl}$, an inhibitor of GSK3 $\beta$ (24), markedly enhanced VEGF release stimulated by FGF-2 in these cells. Additionally, the FGF-2-stimulated VEGF release was amplified by SB216763, a specific inhibitor of GSK3 $\beta$ (26). Therefore, our findings suggest that Wnt3a augments the FGF-2-stimulated VEGF release via the canonical $\mathrm{Wnt} / \beta$-catenin signaling pathway in osteoblast-like MC3T3-E1 cells. In osteoblasts (8), FGF-2-reportedly induces the expression of VEGF mRNA. Furthermore, we demonstrated that Wnt3a as well as SB216763 increased the FGF-2-induced levels of VEGF mRNA. Taking our findings into account, it is most likely that Wnt3a up-regulates the FGF-2-induced VEGF synthesis via the canonical Wnt/ $\beta$-catenin signaling in osteoblasts.

Regarding the signaling system in FGF-2-stimulated VEGF release in osteoblasts, we have previously reported that among the MAP kinase superfamily (11), p44/p42 MAP kinase and SAPK/JNK act as positive regulators, whereas p38 MAP kinase negatively regulates the VEGF release in MC3T3-E1 cells $(9,10)$. It is generally known that the MAP kinase superfamily such as the p44/p42 MAP kinase, the SAPK/JNK and the p38 MAP kinase act as central elements used by mammalian cells to transduce the various extracellular messages (11). However, Wnt3a did not reduce the FGF-2-induced phosphorylation levels of p44/p42 MAP kinase or SAPK/JNK. Therefore, it seems unlikely that Wnt3a acts at a point upstream of the p44/p42 MAP kinase or SAPK/ JNK in the regulation of VEGF synthesis induced by FGF-2 in these cells. Additionally, we have demonstrated that the inhibition of Akt and p70 S6 kinase results in enhancement of the FGF-2-stimulated VEGF release in osteoblast-like MC3T3-E1 cells $(27,28)$. Thus, we next investigated the relationship between the Wnt pathway and these kinases in the FGF-2stimulated VEGF synthesis in MC3T3-E1 cells. However, the FGF-2-induced phosphorylation levels of Akt or p70 S6 kinase was not affected by Wnt3a. Based on these findings, it seems unlikely that Wnt3a affects the FGF-2-stimulated VEGF synthesis via the modulation of Akt or p70 S6 kinase in osteoblast-like MC3T3-E1 cells.

In the process of bone remodeling, the expansion of capillary network providing the microvasculature is essential
(3). It is well known that VEGF is a specific mitogen of vascular endothelial cells (1). Therefore, it is speculated that VEGF synthesized by osteoblasts acts as a potent intercellular mediator between osteoblasts and vascular endothelial cells. Moreover, VEGF is reportedly implicated in trabecular bone formation and expansion of the hypertrophic chondrocyte zone in the epiphyseal growth plate of mouse (30), suggesting the physiological significance of VEGF in bone metabolism. On the other hand, it is well recognized that the bone is a specialized connective tissue with a capacity to regenerate and repair itself. The localized activation of signaling cascades is required for the bone regeneration and fracture healing (31). It has been reported that osteoblasts synthesize Wnt proteins and the Wnt/ $\beta$-catenin signaling pathway has an anabolic role in bone metabolism (14). During bone fracture repair, the Wnt signaling is activated and induces bone regeneration, resulting in the increase of bone mass (14). In addition, it is well known that FGF-2 produced by osteoblasts is accumulated in the extracellular matrix of bone (5). FGF-2 expression in osteoblasts is reportedly up-regulated during fracture repair (6). In the present study, we showed that Wnt3a augmented the FGF-2-stimulated VEGF synthesis in osteoblast-like MC3T3-E1 cells. Taking our results into account as a whole, it is probable that VEGF synthesized by the combination of Wnt3a and FGF-2 in osteoblasts plays a pivotal role through up-regulating the capillary endothelial cells proliferation in the process of bone remodeling, especially bone fracture repair. Regulation of the canonical Wnt/ $\beta$-catenin pathway and the FGF-2 signaling pathway in osteoblasts might provide new therapeutic aspects of bone fracture repair and metabolic bone diseases such as osteoporosis. Further investigations are necessary to clarify the detailed role of the Wnt signaling in bone metabolism.

In conclusion, our results strongly suggest that Wnt3a enhances FGF-2-stimulated VEGF synthesis via activation of the canonical Wnt/ $\beta$-catenin signaling pathway in osteoblasts.

\section{Acknowledgements}

We are very grateful to Mrs. Yoko Kawamura for her skillful technical assistance. This investigation was supported in part by Grant-in-Aid for Scientific Research (19591042) from the Ministry of Education, Science, Sports and Culture of Japan, the Foundation for Growth Science, the research grants for longevity sciences (21A-1, 21A-4 and 21A-22) from the Ministry of Health, Labour and Welfare of Japan.

\section{References}

1. Ferrara N: Vascular endothelial growth factor: basic science and clinical progress. Endocr Rev 25: 581-611, 2004.

2. Karsenty G and Wagner EF: Reaching a genetic and molecular understanding of skeletal development. Dev Cell 2: 389-406, 2002.

3. Erlebacher A, Filvaroff EH, Gitelman SE and Derynck R: Toward a molecular understanding of skeletal development. Cell 80: 371-378, 1995.

4. Zelzer E and Olsen BR: Multiple roles of vascular endothelial growth factor (VEGF) in skeletal development, growth, and repair. Curr Top Dev Biol 65: 169-187, 2005.

5. Baylink DJ, Finkleman RD and Mohan S: Growth factor to stimulate bone formation. J Bone Miner Res 8: S565-S572, 1993. 
6. Bolander ME: Regulation of fracture repair by growth factors stimulate tyrosine kinase activity in vivo. Proc Soc Exp Biol Med 200: 165-170, 1992.

7. Marie PJ: Fibroblast growth factor signaling controlling osteoblast differentiation. Gene 316: 23-32, 2003.

8. Saadeh PB, Mehrara BJ, Steinbrech DS, Spector JA, Greenwald JA, Chin GS, Ueno H, Gittes GK and Longaker MT: Mechanisms of fibroblast growth factor-2 modulation of vascular endothelial growth factor expression by osteoblastic cells. Endocrinology 141 2075-2083, 2000.

9. Tokuda $\mathrm{H}$, Kozawa $\mathrm{O}$ and Uematsu T: Basic fibroblast growth factor stimulates vascular endothelial growth factor release in osteoblasts: divergent regulation by $\mathrm{p} 44 / \mathrm{p} 42$ mitogen-activated protein kinase and p38 mitogen-activated protein kinase. J Bone Miner Res 15: 2371-2379, 2000.

10. Tokuda H, Hirade K, Wang X, Oiso Y and Kozawa O: Involvement of SAPK/JNK in basic fibroblast growth factor-induced VEGF release in osteoblasts. J Endocrinol 177: 101-107, 2003.

11. Kyriakis JM and Avruch J: Mammalian mitogen-activated protein kinase signal transduction pathways activated by stress and inflammation. Physiol Rev 81: 807-869, 2001.

12. Moon RT, Bowerman B, Boutros M and Perrimon N: The promise and perils of Wnt signaling through $\beta$-catenin. Science 296: 1644-1646, 2002.

13. van Amerongen $R$ and Nusse R: Towards an integrated view of Wnt signaling in development. Development 136: 3205-3214, 2009.

14. Milat $\mathrm{F}$ and Wah $\mathrm{Ng} \mathrm{K}$ : Is Wnt signaling the final common pathway leading to bone formation? Mol Cel Endocrinol 310: 52-62, 2009.

15. Hu H, Hilton MJ, Tu X, Yu K, Ornitz DM and Long F: Sequential roles of Hedgehog and Wnt signaling in osteoblast development. Development 132: 49-60, 2005.

16. Krishnan V, Bryant HU and Macdougald OA: Regulation of bone mass by Wnt signaling. J Clin Invest 116: 1202-1209, 2006.

17. Rodda SJ and McMahon AP: Distinct roles for hedgehog and canonical Wnt signaling in specification, differentiation and maintenance of osteoblast progenitors. Development 133 : 3231-3244, 2006

18. Almeida M, Han L, Bellido T, Manolagas SC and Kousteni S: Wnt proteins prevent apoptosis of both uncommitted osteoblast progenitors and differentiated osteoblasts by $\beta$-catenin-dependent and -independent signaling cascades involving Src/ERK and phosphatidylinositol 3-kinase/AKT. J Biol Chem 280 41342-41351, 2005.
19. Sudo H, Kodama H, Amagai Y, Yamamoto $\mathrm{S}$ and Kasai S: In vitro differentiation and calcification in a new clonal osteogenic cell line derived from newborn mouse calvaria. J Cell Biol 96: 191-198, 1983.

20. Kozawa O, Tokuda H, Miwa M, Kotoyori J and Oiso Y: Cross-talk regulation between cyclic AMP production and phosphoinositide hydrolysis induced by prostaglandin E2 in osteoblast-like cells. Exp Cell Res 198: 130-134, 1992.

21. Simpson DA, Feeney S, Boyle C and Stitt AW: Retinal VEGF mRNA measured by SYBR green I fluorescence: A versatile approach to quantitative PCR. Mol Vis 6: 178-183, 2000.

22. Kato $\mathrm{K}$, Ito $\mathrm{H}$, Hasegawa $\mathrm{K}$, Inaguma $\mathrm{Y}$, Kozawa $\mathrm{O}$ and Asano $\mathrm{T}$ : Modulation of the stress-induced synthesis of hsp27 and alpha B-crystallin by cyclic AMP in C6 rat glioma cells. J Neurochem 66: 946-950, 1996.

23. Laemmli UK: Cleavage of structural proteins during the assembly of the head of bacteriophage T4. Nature 227: 680-685, 1970.

24. Stambolic V, Ruel L and Woodgett JR: Lithium inhibits glycogen synthase kinase- 3 activity and mimics wingless signaling in intact cells. Curr Biol 6: 1664-1668, 1996.

25. Case N, Ma M, Sen B, Xie Z, Gross TS and Rubin J: $\beta$-catenin levels influence rapid mechanical responses in osteoblasts. J Biol Chem 283: 29196-29205, 2008.

26. Carmichael J, Sugars KL, Bao YP and Rubinsztein DC Glycogen synthase kinase- $3 \beta$ inhibitors prevent cellular polyglutamine toxicity caused by the Huntington's disease mutation. J Biol Chem 277: 33791-33798, 2002.

27. Takai S, Tokuda H, Hanai Y, Harada A, Yasuda E, MatsushimaNishiwaki R, Kato H, Ogura S, Ohta T and Kozawa O: Negative regulation by p70 S6 kinase of FGF-2-induced VEGF release through stress-activated protein kinase/c-Jun N-terminal kinase in osteoblasts. J Bone Miner Res 22: 337-346, 2007.

28. Takai S, Tokuda H, Hanai Y and Kozawa O: Activation of phosphatidylinositol 3-kinase/Akt limits FGF-2-induced VEGF release in osteoblasts. Mol Cell Endocrinol 267: 46-54, 2007.

29. Chung YS, Baylink DJ, Srivastava AK, Amaar Y, Tapia B, Kasukawa Y and Mohan S: Effects of secreted frizzled-related protein 3 on osteoblasts in vitro. J Bone Miner Res 19: 1395-1402, 2004.

30. Gerber HP, Vu TH, Ryan AM, Kowalski J, Werb Z and Ferrara N: VEGF couples hypertrophic cartilage remodeling, ossification and angiogenesis during endochondral bone formation. Nature Med 5: 623-628, 1999.

31. Secreto FJ, Hoeppner LH and Westendorf JJ: Wnt signaling during fracture repair. Curr Osteoporos Res 7: 64-69, 2009. 